\title{
Automatic determination of complex constants of piezoelectric lossy materials in the radial mode
}

\author{
C Alemany†, A M Gonzálezł, L Pardo†, B Jiménez†, \\ F Carmonat and $J$ Mendiolat \\ † Instituto de Ciencia de Materiales (sede A) CSIC, Serrano 144, 28006 Madrid, \\ Spain \\ † ETSI Telecomunicación (UPM), Ciudad Universitaria, 28040 Madrid, Spain
}

Received 5 December 1994

\begin{abstract}
An iterative automatic method is described for the characterization of lossy piezoelectric materials in the radial resonance mode based on the use of the more general expression for the complex admittance. From the experimental data of $Y$ at four adequately selected frequencies, the constants of the material are determined with the necessary accuracy to reproduce the piezoelectric behaviour of the sample around resonance. The IEEE-176 Standard procedure has been automatized for the initial estimation of the real parts of the elastic constants. The method is applicable even to those materials in which said standard does not allow one to determine the piezoelectric constants.
\end{abstract}

\section{Introduction}

In a previous paper [1], an iterative method for the piezoelectric characterization of materials with four different shapes was reported. With this method it is possible to determine both real and imaginary parts for the whole set of dielectric, elastic and piezoelectric constants [2] in piezoelectric ceramics, polymers and composites. One of the fundamental features of these materials is that they show non-negligible losses, whose expression by means of complex coefficients was introduced by Holland [3] and is now generally accepted.

The use of four geometries for the complete piezoelectric characterization does not permit, however, the direct comparison of the results that are obtained, because for each geometry the polarization process is necessarily different. The materials are isotropic to begin with, and it is the polarization process that brings forth their piezoelectric properties. Therefore, it cannot be guaranteed that, when shapes and polarization processes are different, one is strictly dealing with the same material.

It is therefore essential to determine the constants with the shapes and states of polarization in which the materials are going to be used. One of the more frequently used geometries is that of an axially polarized disc, due to the relative ease of mechanization and polarization processes and its many applications. The characterization of the thickness mode of vibration has already been described in detail in [1]. It is also possible to determine complex coefficients for the radial mode of vibration; but the circular shape imposes some important mathematical differences, and the algorithm must be modified in order to obtain more accurate results.

The expressions for the characterization of the resonance in the radial mode are due to Meitzler et al [4] and have been adopted by the IEEE Standard on Piezoelectricity [5], but these authors apply the method to materials in which losses are negligible, and therefore obtain only real coefficients. The introduction of complex coefficients for the radial mode presents some mathematical difficulties. Sherrit et al [6] propose the determination of the parameters of the mode by a noniterative method. Our proposal is based, on the contrary, on an adaptation of the method described for the rest of the geometries [1], introducing complex expressions for the modified Bessel coefficients, and changing the iterative calculation algorithm for its correct application to circular geometry. The aim is to reproduce, as perfectly as possible, the resonance and anti-resonance profiles simultaneously, both in the fundamental mode and in its first overtone.

\section{The experimental method}

The piezoelectric ceramics with which the method has been tested belong to the catalogue of commercial materials manufactured by Ferroperm Ltd, and were machined to the required geometry (thin discs) and thickness-poled. The results reported here correspond to three samples, PZ27 (lead zirconate titanate), PZ35 (lead metaniobate) and PZ45 (bismuth niobate); chosen to cover a wide range of values of the planar piezoelectric coupling factor and of the mechanical quality factor. 
The measurement technique is the same as that described in our previous paper [1], and consists of provoking resonance at room temperature by electrical stimulation with $1 \mathrm{~V}$ RMS by means of a HP-4192A LF impedance analyser, computer-controlled through a GPIB-PCIIA interface board (National Instruments). The program for data acquisition and processing, in Microsoft QuickBASIC 4.5, using double-precision variables, is available from the authors without charge.

Frequency sweeps were carried out covering two intervals in which fundamental and first overtone resonance and anti-resonance appear, measuring the absolute electric admittance $|Y|$ and its phase angle $\theta$, and storing them in the computer and in the data files. From these data, the associated values of conductance $G=|Y| \cos \theta$, susceptance $B=|Y| \sin \theta$, resistance $R=\cos \theta /|Y|$ and reactance $X=-\sin \theta /|Y|$ were generated, both for the calculations and for the representation of the experimental resonance profiles.

From the positions of the maxima of $G$ and $R$ in the fundamental mode, the frequencies of resonance, $f_{\mathrm{s}}$, and anti-resonance, $f_{\mathrm{p}}$ were inferred, and the corresponding admittance values stored. The two resonance bands were then swept with at least 20 points. The experimental half widths, $\Delta f_{\mathrm{s}}$ (the difference in frequencies between minimum and maximum of $B$ around $f_{\mathrm{s}}$ ) and $\Delta f_{\mathrm{p}}$ (the difference in frequencies between extremal values of $X$ around $f_{p}$ ) were thus determined with precision by leastsquares fits. The first overtone resonance frequency, $f_{2 s}$. was obtained from admittance data. This is the only parameter not related to the fundamental resonance taking part in the characterization process.

The computer demands from the analyser, during the iteration process, new measurements of $|Y|$ and $\theta$ for two other frequency values, $f_{1}$ and $f_{2}$ (see below). The experimental profiles obtained in the two initial sweeps do not directly take part in the process, but are also stored so as to check the agreement a posteriori with the profiles calculated from the values of the constants obtained in the characterization.

In the acquisition of experimental data, and especially from materials with a low piezoelectric yield, it is very important to be careful with the conditions under which measurements are performed, so that they stick, as closely as possible, to the boundary conditions required by theory. Thus, samples must be free from external pressure and electrical contacts should not be made with springs, but rather by the simple friction of a conducting wire with the electrode. Luckily, radial resonance of ceramic discs is usually very clean, and it is relatively easy to detect when the contact is poor or the sample not properly positioned.

\section{Calculation procedures}

\subsection{Generalities}

The electrical admittance as a function of frequency of a ceramic, plastic or composite thin disc, polarized in the direction of its principal axis and vibrating in the radial mode, is obtained from the linear equations of piezoelectricity. The expression, in which the three contributions (dielectric, piezoelectric and elastic) are clearly separated, is

$$
\begin{aligned}
& Y=i \frac{2 \pi^{2} f a^{2}}{t} \\
& \times\left(\epsilon_{0} \epsilon_{33}^{\mathrm{T}}+2 d_{31}^{2} \frac{c_{11}^{\mathrm{p}}}{\frac{1}{2-\Im_{1}\left[2 \pi f a\left(\rho / c_{11}^{\mathrm{p}}\right)^{1 / 2}\right]}-\frac{1}{1+\sigma^{\mathrm{p}}}}\right)
\end{aligned}
$$

derived from equation (117) of the IEEE Standard [5] by replacing the planar coupling factor by the well-known relations

$$
k_{\mathrm{p}}^{2}=\frac{2 d_{31}^{2}}{\epsilon_{0} \epsilon_{33}^{\mathrm{T}}\left(s_{11}^{\mathrm{E}}+s_{12}^{\mathrm{E}}\right)}
$$

and

$$
\begin{gathered}
\sigma^{\mathrm{p}}=-s_{12}^{\mathrm{E}} / s_{11}^{\mathrm{E}} \\
c_{11}^{\mathrm{P}}=\frac{s_{11}^{\mathrm{E}}}{\left(s_{11}^{\mathrm{E}}\right)^{2}-\left(s_{12}^{\mathrm{E}}\right)^{2}} .
\end{gathered}
$$

Parameters $\rho$ (density), $a$ (radius), $t$ (thickness), the universal constant $\epsilon_{0}$ and the oscillation frequency $f$ are real numbers, but the rest of the variables are complex quantities: the piezoelectric constant, $d_{31}$, the relative permittivity, $\epsilon_{33}^{\mathrm{T}}$, Poisson's planar ratio, $\sigma^{\mathrm{p}}$, and $c_{11}^{\mathrm{p}}$. The last two are related to the standard compliance constants $s_{11}^{E}$ and $s_{12}^{E}$ by the previous expressions.

$\Im_{1}$ is also a complex function of a complex variable, called Onoe's function by Meitzler et al [4], and defined by

$$
\Im_{1}(z)=\frac{z J_{0}(z)}{J_{1}(z)}
$$

where $J_{0}$ and $J_{1}$ are Bessel functions of the first kind, of zeroth and first orders, of the argument

$$
z=2 \pi f a\left(\rho / c_{11}^{\mathrm{p}}\right)^{1 / 2} .
$$

Other constants that may be obtained with the general equations of piezoelectricity from the previous parameters are $s_{66}^{\mathrm{E}}, g_{31}, s_{11}^{\mathrm{D}}$ and $s_{12}^{\mathrm{D}}$, and from them, by analogy with equations (3), the elastic stiffness at constant $D,\left(c_{11}^{\mathrm{p}}\right)^{D}$.

The standard method assumes that there is a resonance when the admittance tends to infinity, thus implying that the denominator in equation (1) is zero, that is

$$
1-\sigma^{p}-\Im\left(\eta_{1}\right)=0
$$

where $\eta_{1}$ is the value of $z$ obtained from (4) for the fundamental resonance frequency. (For the first overtone frequency, the value of the argument is $\eta_{2}$.)

In practice, and mainly in lossy materials, such as piezoelectric ceramics, the admittance $Y$ is far from infinity, and this requires the adoption of alternative, and more precise, characterization procedures. 
Except for the admittance $Y=G+\mathrm{i} B$, complex quantities have been written, following Holland [3], as

$$
\text { real part }-\mathrm{i} \times \text { imaginary part. }
$$

The elements of the diagonal of the elastic-piezodielectric matrix are thus forced to have positive imaginary parts, and this, for the resonance in the radial mode, amounts to

$$
\begin{aligned}
& \left(\epsilon_{33}^{\mathrm{T}}\right)^{\prime \prime} \geqslant 0 \\
& \left(s_{11}^{\mathrm{E}}\right)^{\prime \prime} \geqslant 0 .
\end{aligned}
$$

Other conditions that, according to Holland, should hold are

$$
\begin{aligned}
& \left(s_{11}^{\mathrm{E}}\right)^{\prime \prime} \geqslant \mid\left(s_{12}^{\mathrm{E}}\right)^{\prime \prime} ! \\
& \left(s_{11}^{\mathrm{E}}\right)^{\prime \prime} \epsilon_{0}\left(\epsilon_{33}^{\mathrm{T}}\right)^{\prime \prime} \geqslant\left(d_{31}^{\prime \prime}\right)^{2} .
\end{aligned}
$$

All these may be used as first criteria to evaluate the adequacy of the method.

For each parameter it is possible to define a quality factor, $Q$, as the absolute value of the ratio of the real component to the imaginary component, although in this paper we shall only use $Q_{\mathrm{m}}$, the quality factor associated with the mechanical constants.

The real and imaginary parts of the experimental admittance and impedance bands around the fundamental resonance have been reproduced, using equation (1), with the final values of the characterization parameters. The agreement between experimental and calculated profiles is the second criterion used to evaluate the degree of precision achieved.

Considering the possible dependence on frequency of said parameters, it could be somewhat unrealistic to extend the results of measurements, performed within a small interval of frequencies around the fundamental resonance, to other overtones of the piezoelectric resonance outside that region. However, and this is our third and last criterion of accuracy of the method, we aim at reproducing acceptably the $G$ and $R$ profiles of the first overtone from the calculated values of the parameters (it is considered unnecessary to include $B$ and $X$ ).

\subsection{Getting started}

The constants $d_{31}, \epsilon_{33}^{\mathrm{T}}, \sigma^{\mathrm{p}}$ and $c_{11}^{\mathrm{p}}$ are determined by measuring $Y$ at four different frequencies, adequately chosen around the fundamental resonance, and solving the resulting system of equations of type (1) by an iteration method.

The resonance and anti-resonance frequencies, $f_{\mathrm{s}}$ and $f_{p}$, which correspond to maxima of the $G$ and $R$ bands, are determined in the data acquisition operation at the beginning of the process. They are generally accepted as most representative of the fundamental resonance, at which the electric and elastic energies have extremal values.

The other two frequencies are obtained, as for the rest of the geometries [1], from

$$
f_{1,2}=\frac{f_{\mathrm{s}}}{\left(1 \pm\left|k_{\mathrm{p}}\right|\right)^{1 / 2}}
$$

which is an extension, to this resonance mode, of the formula for the frequencies associated with a maximum piezoelectric energy content [2]. The determination of these two frequencies is the goal of the peripheral iterative loop involved in our method (figure 1). However, the coupling factor $k_{\mathrm{p}}$ is unknown, and an initial estimation of $f_{1}$ and $f_{2}$ is required. We have taken, as initial values for $f_{1}$ and $f_{2}$, the experimental values of the maximum and the minimum of the susceptance $B$ around $f_{s}$, corresponding to balf-height values in the resonance band $f_{2,1}=f_{\mathrm{s}} \pm \Delta f_{\mathrm{s}}$.

\subsection{Initial estimation of the elastic constants}

Constants $\sigma^{\mathrm{p}}$ and $c_{11}^{\mathrm{p}}$ both participate in the elastic term of the admittance (equation (1)), which causes a certain indeterminacy because, for a given frequency, the contribution of said term may be the same for widely (and wildly) different values of the constants. A good initial estimation for them is therefore needed. For the real parts we have adopted the values provided by the IEEE Standard characterization method, which, given the ease of calculation provided by present computers, has been rigorously implemented and included in our program, without linear interpolations or polynomial fits to the data of table 12 of the IEEE Standard.

The problem is just to solve a system of two transcendental equations with two unknowns, $\sigma^{\mathrm{P}}$ and $c_{11}^{\mathrm{P}}$, of the form

$$
\begin{aligned}
& 1-\sigma^{\mathrm{p}}-\Im\left(\eta_{1}\right)=0 \\
& 1-\sigma^{\mathrm{P}}-\Im\left(\eta_{2}\right)=0
\end{aligned}
$$

which are not linear and thus require an iterative process. Beginning with $\sigma^{\mathfrak{p}}=0$, the argument $\eta_{1}$ is obtained by calculating the inverse function of $\Im_{1}$ as described in the appendix. Next, the argument $\eta_{2}$ is determined from $\eta_{2}=\eta_{1}\left(f_{2 s} / f_{s}\right)$ and then, using the direct calculation procedure of $\widetilde{\Im}_{1}$, we obtain a new value of $\sigma^{P}$. This is the only place in which the first overtone frequency $f_{2 s}$ takes part. In the rest of the process only experimental data around the fundamental resonance are used.

If the absolute value of the difference between two successive values of $\sigma^{p}$ is higher than $10^{-8}$, then $\sigma^{p}$ is increased by the tenth of such difference, to ensure convergence. If not, we have arrived at the initial estimation of the real part of $\sigma^{\mathrm{p}}$. From the last argument $\eta_{1}$ obtained, and from the frequency $f_{s}$, the value given by

$$
\left(c_{11}^{\mathrm{p}}\right)^{\prime}=\left(\frac{2 a \pi f_{\mathrm{s}}}{\eta_{\mathrm{I}}}\right)^{2} \rho
$$

is taken as initial estimation of the real part of $c_{11}^{\mathrm{p}}$.

This automatic implementation of the IEEE Standard procedure is only applied to real constants, and considers as zero all the contributions of the imaginary parts mentioned in the sub-routines of the appendix. We next turn to the estimation of the imaginary parts of the two mechanical constants.

The initial estimation of the imaginary part of $\sigma^{p}$ is taken as zero, which is equivalent to considering, 


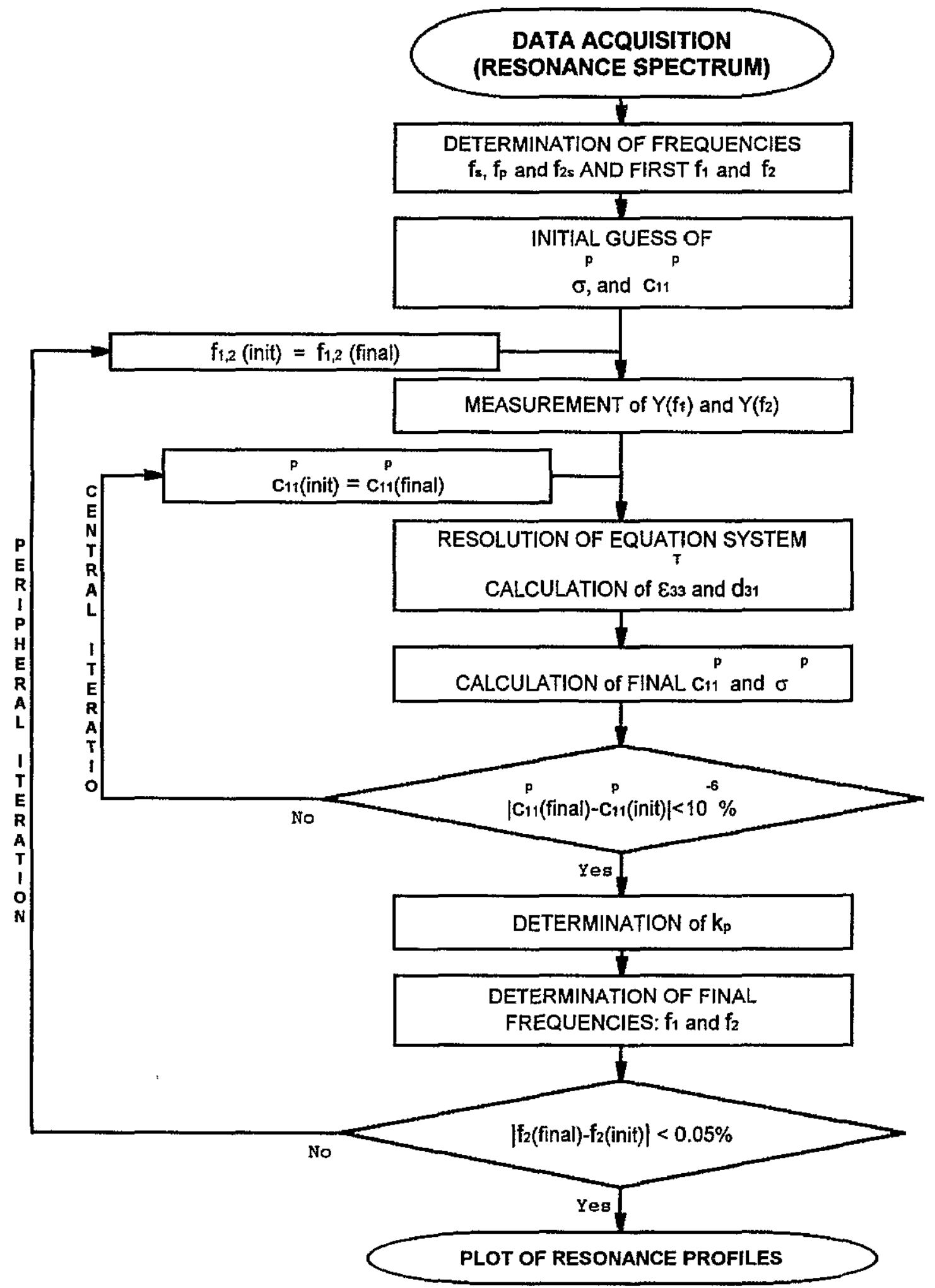

Figure 1. The data-acquisition and calculation process.

according to equations (3), that the quality factors associated with the compliance constants $s_{11}^{\mathrm{E}}$ and $s_{12}^{\mathrm{E}}$ and the stiffness constant $c_{11}^{p}$ are equal. Next, the imaginary part of $c_{11}^{\mathrm{p}}$ is initially estimated from the experimental half-width of the resonance band, $\Delta f_{\mathrm{s}}$, by

$$
\left(c_{11}^{\mathrm{p}}\right)^{\prime \prime}=-\frac{\Delta f_{\mathrm{s}}}{f_{\mathrm{s}}}\left(c_{11}^{\mathrm{p}}\right)^{\prime}
$$

\subsection{The iterative process}

With these values for $\sigma^{\mathrm{p}}$ and $c_{11}^{\mathrm{p}}$, with the experimental values of $Y$ for the initial frequencies $f_{1}$ and $f_{2}$, and with the direct calculation of $\Im_{1}(z)$ at these two frequencies (see the appendix), the piezoelectric coefficient $d_{31}$ and the relative permittivity at constant stress $\epsilon_{33}^{\mathrm{T}}$ are 
determined from a system of two equations of type (1).

From the measurement of the admittance at $f_{\mathrm{p}}$, from the value of $\Im_{1}(z)$ at this frequency, and from the rest of the parameters, a better value of $d_{31}$ is then calculated; but when there is no observable difference between $f_{s}$ and $f_{\mathrm{p}}$ (such as in the case of PZ35 samples) this step is omitted because, apart from not providing a better value, it would not be compatible with the next step and the calculation would be aborted.

Finally, from the experimental value of $Y$ at $f_{\mathrm{s}}$, better approximations to the unknowns $\sigma^{\mathrm{p}}$ and $c_{11}^{\mathrm{p}}$ are obtained. To begin with, a new $c_{11}^{\mathrm{p}}$ is determined from the immediately preceding results for the four constants, just by calculating the complex argument $\eta_{1}$ corresponding to $\Im_{1}\left(\eta_{1}\right)$ by applying once more the inverse calculation procedure described in the appendix. From this argument a better value is immediately obtained for the stiffness constant from equation (4). Then, still at this frequency $f_{\mathrm{s}}$, with the new values of $c_{11}^{\mathrm{p}}$ and $\Im_{1}\left(\eta_{1}\right)$, and the previous ones of $d_{31}$ and $\epsilon_{33}^{\mathrm{T}}$, a better $\sigma^{\mathrm{P}}$ is obtained.

A central iteration loop has been completed, and the process is repeated, as shown in figure 1 , until the final and the initial values of $c_{11}^{\mathrm{p}}$ differ by less than $10^{-6} \%$. Two new values for frequencies $f_{1}$ and $f_{2}$ are then calculated with equations (2) and (8). If the difference between the initial and the final $f_{2}$ is higher than $\pm 0.05 \%$, then the central iteration loop is once more taken, after measuring $Y$ at the new $f_{1}$ and $f_{2}$ that have just been calculated and taking as initial values the last values of $\sigma^{\mathrm{p}}$ and $c_{11}^{\mathrm{p}}$.

The application of this method showed that, as a rule, the initial and final values of $f_{1}$ and $f_{2}$ are quite different. On the other hand, the coupling factor turns out to be quite stable from the outset. We thus decided that the first central iteration should consist of just one step, because this is sufficient to obtain a good value of $k_{\mathrm{p}}$ so as to get (via equation (8)) into the peripheral iteration, which repeats some $2-5$ times before all the cut-off criteria are fulfilled. The number of steps of the central iteration is in the range $5-50$; therefore, the total number of steps varies in the range 5-200, and thus the whole process, including intermediate measurements in $f_{1}$ and $f_{2}$ takes place within a few minutes.

\section{Results and discussion}

The method has been tested with a wide range of ceramic discs, with values of $k_{\mathrm{p}}$ in the range $2-62 \%$, and with $Q$ up to 3500 , and has proved its efficiency with all the samples, for whatever coupling factors and mechanical losses, even in such extreme cases as those in which the small resolution between resonance and antiresonance peaks makes difficult, if not impossible, the complete characterization by the procedure suggested by the IEEE Standard. We have been able to determine all the constants that are characteristic of the radial mode, around its fundamental resonance, by simply measuring real and imaginary parts of the admittance. Thus, it has not been necessary to approach the values of the constants with measurements at different frequencies, as is usually done in the case of $\epsilon_{33}^{\mathrm{T}}$. The first criterion of evaluation, concerning the sign and the modulus of the imaginary part of the constants (equations (6) and (7)), is satisfied for all the samples that have been studied. Three of them, very different from each other, have been chosen for the following discussion.

In figures $2(a)$ and $(b)$ we present experimental results for the fundamental resonance of a disc resonator (PZ27 Ferroperm with $t=1.0 \mathrm{~mm}, a=10.0 \mathrm{~mm}$ and $\rho=7.70 \mathrm{~g} \mathrm{~cm}^{-3}$ ), together with continuous curves for $G$ and $R$ (figure 2(a)) and for $B$ and $X$ (figure $2(b)$ ), which were calculated using equation (1) with the final values of the constants determined by the iterative method that has been described. Making a sweep around a frequency between two and three times higher than the fundamental resonance, it is possible to generate $G$ and $R$ for the first overtone, and this is what we have in figure $2(c)$, together with the experimental data. The final values of the constants are given in table 1 , in which the results that would be obtained using the IEEE Standard characterization method are also shown. Values for $f_{\mathrm{s}} / \Delta f_{\mathrm{s}}$ and $f_{\mathrm{p}} / \Delta f_{\mathrm{p}}$ in the 'iterative' column were computed using the calculated maxima of $G$ and $R$ and maxima and minima of $B$ and $X$. Thus, $f_{\mathrm{s}} / \Delta f_{\mathrm{s}}=f_{G \text { max }} /\left(f_{B \text { min }}-f_{B \text { max }}\right)$ and $f_{\mathrm{p}} / \Delta f_{\mathrm{p}}=f_{R \max } /\left(f_{X \min }-f_{X \max }\right)$.

The samples of this group may be considered as typical lead zirconate titanate ceramics with high planar coupling factors and moderate mechanical quality factors. In them, the reproduction of the fundamental resonance is next to perfect (figures $2(a)$ and $(b)$ ), and the reproduction of the first overtone is quite acceptable (figure $2(c)$ ). It is to be noted that, for this resonance, only the frequency of the maximum of $G$ took part in the characterization process, and that any minor variation with frequency of the constants would justify the small differences for this overtone between the experimental data and the calculated curve.

Figures $3(a)-(c)$ show curves of the same type for another resonant disc (PZ45 Ferroperm with $t=$ $0.50 \mathrm{~mm}, a=5.0 \mathrm{~mm}$ and $\rho=7.20 \mathrm{~g} \mathrm{~cm}^{-3}$ ) and table 2 summarizes the final values for the constants and, for comparison, results obtained by applying the IEEE Standard. The agreement is excellent because high and narrow $G$ and $R$ bands agree very well with the hypothesis made in the Standard of infinite admittance at resonance and zero admittance at antiresonance. However, our characterization leads to a small discrepancy in the reproduction of the tail, for frequencies under $f_{\mathrm{s}}$ and $f_{\mathrm{p}}$, of the fundamental resonance profiles (figure $3(a)$ ). This distortion disappears altogether, and the reproduction becomes very good when measurements are repeated modifying the oscillator level of the analyser from $1 \mathrm{~V}$ RMS (default value) to $0.1 \mathrm{~V}$ RMS. The constants remain almost unaltered, except for $d_{31}$, which decreases by $6 \%$. This effect could be associated, according to Woollett and Le Blanc [7], with ferroelectric nonlinearities produced by 

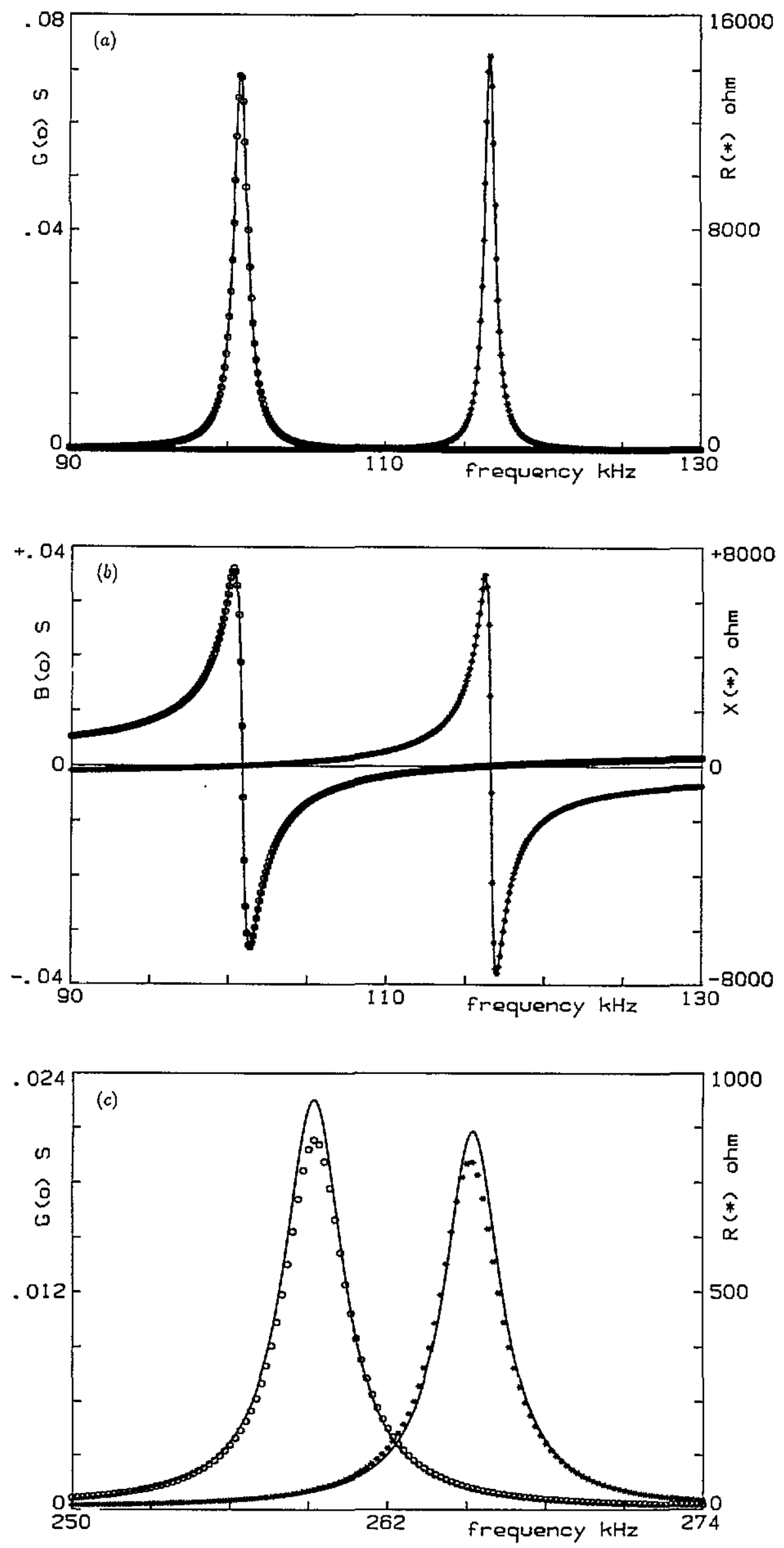

Figure 2. Radial resonance and anti-resonance profiles of the PZ27 sample: (a) the fundamental mode: real parts; (b) the fundamental mode: imaginary parts; and (c) the first overtone. 
Table 1. PZ27 sample characterization.

\begin{tabular}{|c|c|c|c|}
\hline & Experimental & Standard & Iterative \\
\hline $\begin{array}{l}f_{s}(\mathrm{kHz}) \\
f_{\mathrm{p}}(\mathrm{kHz}) \\
C(\mathrm{pF} \text { at }+\mathrm{kHz})\end{array}$ & $\begin{array}{l}100.8 \\
116.6 \\
4808\end{array}$ & & \\
\hline Number of iterations & & & 6 \\
\hline $\begin{array}{l}k_{p} \\
\sigma^{p}\end{array}$ & & $\begin{array}{l}0.5718 \\
0.3899\end{array}$ & $\begin{array}{l}0.5718-i 0.0034 \\
0.3904+i 0.00002\end{array}$ \\
\hline $\begin{array}{l}f_{s} / \Delta f_{s} \\
c_{11}^{p}\left(10^{10} \mathrm{~N} \mathrm{~m}^{-2}\right)\end{array}$ & 94.86 & 6.9894 & $\begin{array}{l}94.53 \\
6.9876+i 0.0745\end{array}$ \\
\hline$Q_{\mathrm{m}}\left(c_{11}^{\mathrm{p}}\right)$ & & & 93.85 \\
\hline $\begin{array}{l}s_{11}^{E}\left(10^{-12} m^{2} N^{-1}\right) \\
Q_{m}\left(s_{11}^{E}\right)\end{array}$ & & 16.8726 & $\begin{array}{l}16.8826-j 0.1796 \\
94.00\end{array}$ \\
\hline $\begin{array}{l}s_{12}^{E}\left(10^{-12} m^{2} N^{-1}\right) \\
Q_{m}\left(s_{12}^{E}\right)\end{array}$ & & -6.5790 & $\begin{array}{l}-6.5910+i 0.0698 \\
94.42\end{array}$ \\
\hline$\epsilon_{33}^{\top}$ & & 1730.66 & $1651.12-\mathrm{i} 28.76$ \\
\hline $\begin{array}{l}d_{31}\left(10^{-12} \mathrm{CN}^{-1}\right) \\
f_{p} / \Delta f_{p}\end{array}$ & 147.55 & -160.465 & $\begin{array}{l}-156.704+i 3.116 \\
149.85\end{array}$ \\
\hline$\left(c_{11}^{\mathrm{P}}\right)^{D}\left(10^{10} \mathrm{~N} \mathrm{~m}^{-2}\right)$ & & 9.3488 & $9.3473+i 0.0587$ \\
\hline $\begin{array}{l}Q_{m}\left(\left(c_{11}^{P}\right)^{D}\right) \\
s_{11}^{D}\left(10^{-12} \mathrm{~m}^{2} \mathrm{~N}^{-1}\right) \\
Q_{m}\left(s_{11}^{D}\right)\end{array}$ & & 15.1900 & $\begin{array}{l}159.29 \\
15.2005-10.1420 \\
107.04\end{array}$ \\
\hline $\begin{array}{l}s_{12}^{D}\left(10^{-12} \mathrm{~m}^{2} \mathrm{~N}^{-1}\right) \\
Q_{\mathrm{m}}\left(s_{12}^{\mathrm{D}}\right)\end{array}$ & & -8.2617 & $\begin{array}{l}-8.2731+10.1074 \\
\quad 77.03\end{array}$ \\
\hline
\end{tabular}

Table 2. PZ45 sample characterization.

\begin{tabular}{|c|c|c|c|}
\hline & Experimental & Standard & Iterative \\
\hline $\begin{array}{l}f_{\mathrm{s}}(\mathrm{kHz}) \\
f_{\mathrm{p}}(\mathrm{kHz}) \\
C(\mathrm{pF} \text { at } 1 \mathrm{kHz})\end{array}$ & $\begin{array}{l}276.244 \\
276.316 \\
235.7\end{array}$ & & \\
\hline Number of iterations & & & 15 \\
\hline $\begin{array}{l}k_{p} \\
\sigma^{p}\end{array}$ & & $\begin{array}{l}0.0257 \\
0.2490\end{array}$ & $\begin{array}{l}0.0258+i 0.0001 \\
0.2490+i 0.00000\end{array}$ \\
\hline $\begin{array}{l}f_{s} / \Delta f_{s} \\
c_{i 1}^{p}\left(10^{10} \mathrm{~N} \mathrm{~m}^{-2}\right)\end{array}$ & 3141.19 & 13.3357 & $\begin{array}{l}2897.44 \\
13.3356+10.0046\end{array}$ \\
\hline$Q_{m}\left(c_{11}^{p}\right)$ & & & 2899.63 \\
\hline $\begin{array}{l}s_{11}^{E}\left(10^{-12} \mathrm{~m}^{2} \mathrm{~N}^{-1}\right) \\
Q_{\mathrm{m}}\left(s_{11}^{\mathrm{E}}\right)\end{array}$ & & 7.9942 & $\begin{array}{l}7.9943-i 0.0028 \\
2899.65\end{array}$ \\
\hline $\begin{array}{l}s_{12}^{E}\left(10^{-12} m^{2} N^{-1}\right) \\
Q_{m}\left(s_{12}^{E}\right)\end{array}$ & & -1.9903 & $\begin{array}{l}-1.9905+10.0007 \\
2899.74\end{array}$ \\
\hline$\epsilon_{33}^{\top}$ & & 169.70 & $167.75-10.50$ \\
\hline $\begin{array}{l}d_{31}\left(10^{-12} \mathrm{CN}^{-1}\right) \\
f_{\mathrm{p}} / \Delta f_{\mathrm{p}}\end{array}$ & 2898.46 & -1.7252 & $\begin{array}{l}-1.7190+i 0.0021 \\
2864.35\end{array}$ \\
\hline $\begin{array}{l}\left(c_{11}^{\mathrm{p}}\right)^{D}\left(10^{10} \mathrm{~N} \mathrm{~m}^{-2}\right) \\
Q_{m}\left(\left(c_{1+1}^{\mathrm{p}}\right)^{D}\right)\end{array}$ & & 13.3412 & $\begin{array}{l}13.3412+10.0046 \\
2879.69\end{array}$ \\
\hline $\begin{array}{l}s_{11}^{D}\left(10^{-12} \mathrm{~m}^{2} \mathrm{~N}^{-1}\right) \\
Q_{\mathrm{m}}\left(s_{11}^{\mathrm{D}}\right)\end{array}$ & & 7.9922 & $\begin{array}{l}7.9923-i 0.0028 \\
2887.63\end{array}$ \\
\hline $\begin{array}{l}s_{12}^{\mathrm{D}}\left(10^{-12} \mathrm{~m}^{2} \mathrm{~N}^{-1}\right) \\
Q_{\mathrm{m}}\left(s_{12}^{\mathrm{D}}\right)\end{array}$ & & -1.9923 & $\begin{array}{l}-1.9925+i 0.0007 \\
2948.96\end{array}$ \\
\hline
\end{tabular}

the excitation voltage, and further study of this subject would be inappropriate here.

Finally, figures $4(a)-(c)$ show curves for another disc (PZ35 Ferroperm with $t=0.77 \mathrm{~mm}, a=$ $5.2 \mathrm{~mm}$ and $\rho=5.70 \mathrm{~g} \mathrm{~cm}^{-3}$ ) and in table 3 we have our values for the constants and the best possible characterization following the Standard. In this case the latter is necessarily incomplete, because there is no measurable difference between resonance and anti-resonance frequencies. A least-squares fit to a conic around the experimental maxima of $G$ and $R$ shows that, rather than being coincidental, said 

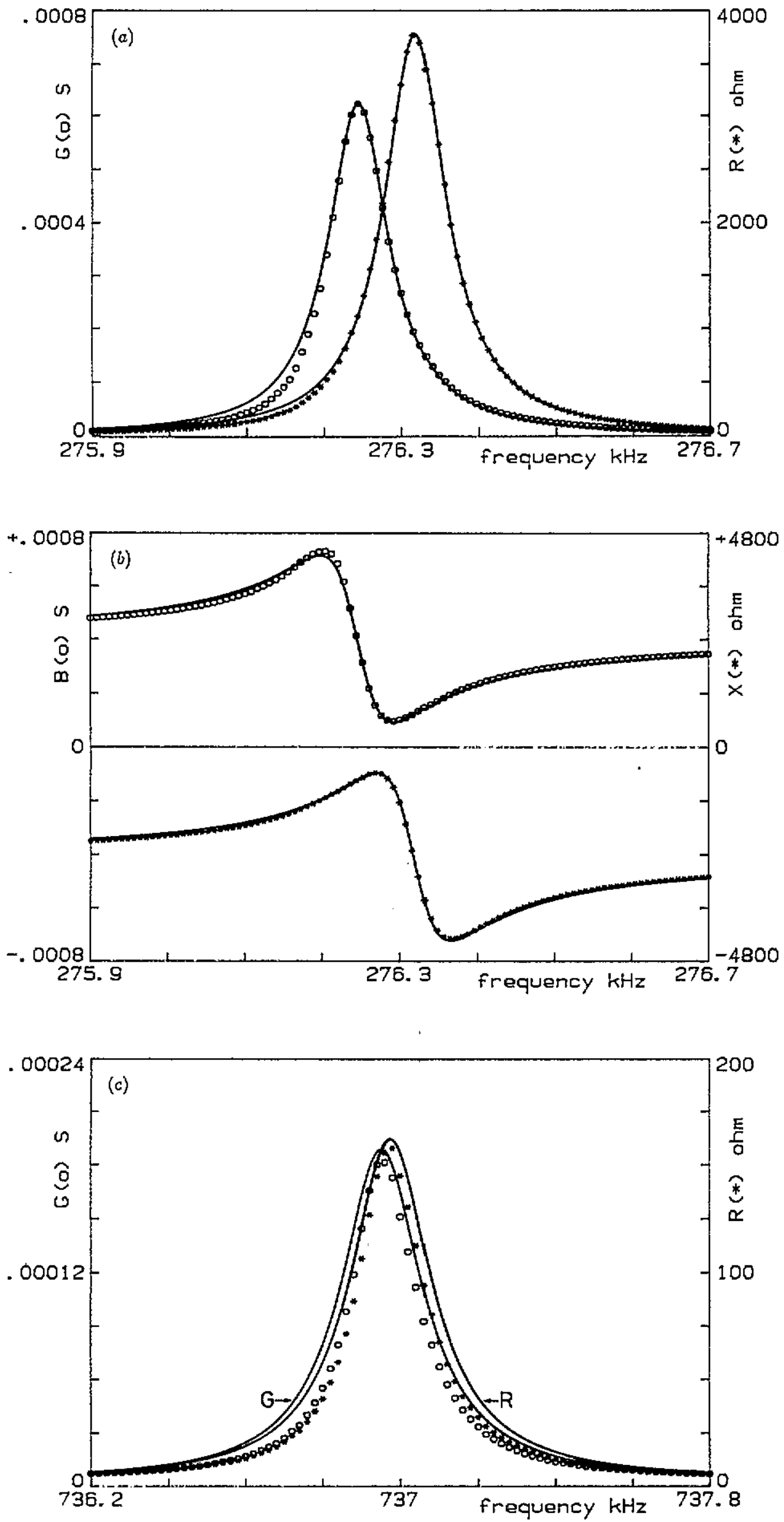

Figure 3. Radial resonance and anti-resonance profiles of the PZ45 sample: (a) the fundamental mode: real parts; $(b)$ the fundamental mode: imaginary parts; and (c) the first overtone. 

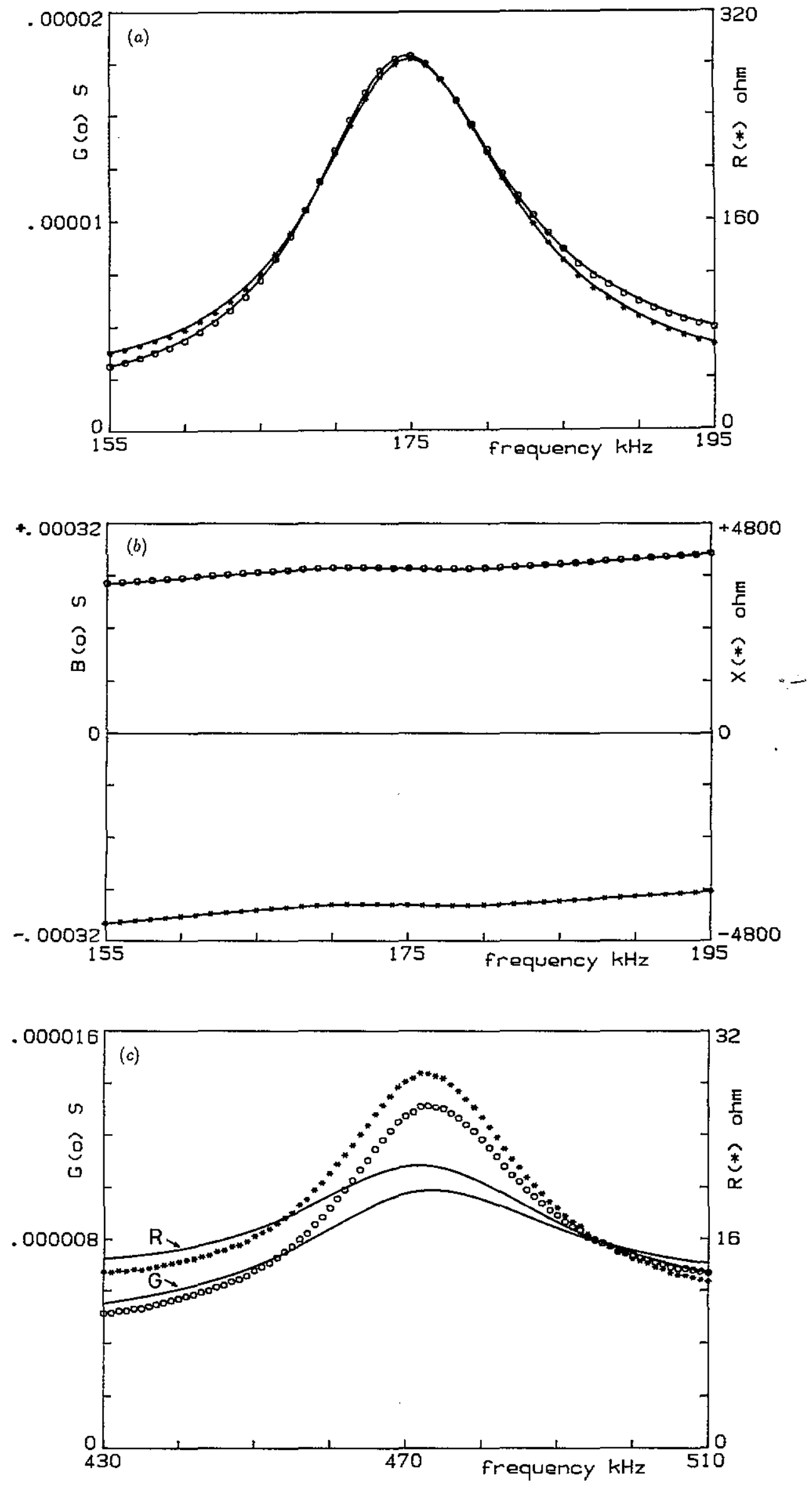

Figure 4. Radial resonance and anti-resonance profiles of the PZ35 sample: (a) the fundamental mode: real parts; $(b)$ the fundamental mode: imaginary parts; and $(c)$ the first overtone. 
Table 3. PZ35 sample characterization.

\begin{tabular}{|c|c|c|c|}
\hline & Experimental & Standard & Iterative \\
\hline $\begin{array}{l}f_{\mathrm{s}}(\mathrm{kHz}) \\
f_{\mathrm{p}}(\mathrm{kHz}) \\
C(\mathrm{pF} \text { at } 1 \mathrm{kHz}) \\
\text { Number of iterations } \\
k_{\mathrm{p}} \\
\sigma^{\mathrm{P}} \\
f_{\mathrm{s}} / \Delta f_{\mathrm{s}} \\
c_{11}^{\mathrm{O}}\left(10^{10} \mathrm{~N} \mathrm{~m}^{-2}\right) \\
Q_{\mathrm{m}}\left(c_{11}^{\mathrm{p}}\right) \\
s_{11}^{\mathrm{E}}\left(10^{-12} \mathrm{~m}^{2} \mathrm{~N}^{-1}\right) \\
Q_{\mathrm{m}}\left(s_{11}^{\mathrm{E}}\right) \\
s_{12}^{\mathrm{E}}\left(10^{-12} \mathrm{~m}^{2} \mathrm{~N}^{-1}\right) \\
Q_{\mathrm{m}}\left(s_{12}^{\mathrm{E}}\right) \\
\epsilon_{33}^{\mathrm{T}} \\
d_{31}\left(10^{-12} \mathrm{CN}^{-1}\right) \\
f_{\mathrm{p}} / \Delta f_{\mathrm{p}} \\
\left(c_{11}^{\mathrm{p}}\right)^{\mathrm{D}}\left(10^{10} \mathrm{~N} \mathrm{~m}^{-2}\right) \\
Q_{\mathrm{m}}\left(\left(c_{11}^{\mathrm{P}}\right)^{D}\right) \\
s_{11}^{\mathrm{D}}\left(10^{-12} \mathrm{~m}^{2} \mathrm{~N}^{-1}\right) \\
Q_{\mathrm{m}}\left(s_{11}^{\mathrm{D}}\right) \\
s_{12}^{\mathrm{D}}\left(10^{-12} \mathrm{~m}^{2} \mathrm{~N}^{-1}\right) \\
Q_{\mathrm{m}}\left(s_{12}^{\mathrm{D}}\right)\end{array}$ & $\begin{array}{l}175 \\
175 \\
234.4\end{array}$ & $\begin{array}{r}0.2039 \\
4.7222 \\
22.0956 \\
-4.5048 \\
240.15\end{array}$ & $\begin{array}{l}6 \\
0.0844+i 0.0032 \\
0.1985-i 0.0011 \\
32.16 \\
4.6813+i 0.4249 \\
11.02 \\
22.0552-i 2.0116 \\
10.96 \\
-4.3755+i 0.4225 \\
10.36 \\
234.36-i 1.68 \\
-11.449+i 0.116 \\
35.22 \\
4.7012+i 0.4283 \\
10.98 \\
21.9919-i 2.0107 \\
10.94 \\
-4.4388+i 0.4233 \\
10.48\end{array}$ \\
\hline
\end{tabular}

frequencies are slightly different and inverted, which is typical of piezoelectrics with high losses. Our method, however, is very good as regards the reproduction of the fundamental resonant mode (figures $4(a)$ and $(b)$ ). It is only fairly good, however, concerning the first overtone (figure $4(c)$ ), because the backgrounds and the frequencies of the peaks are acceptably reproduced, whereas the heights of the peaks differ by some $25 \%$. This is probably due to a marked dependence of $d_{31}$ on frequency. The fact that the frequencies are properly reproduced indicates a small variation in the elastic constants; and the similarity in the backgrounds also suggests a small variation in the dielectric permittivity.

As regards the relation between half-widths and mechanical quality factors, it is clearly seen that, when the planar Poisson ratio is practically a real quantity (PZ27 and PZ45), the quality factors associated with the elastic parameters at constant $E$ are almost equal, and similar, in turn, to the ratio $f_{\mathrm{s}} / \Delta f_{\mathrm{s}}$ of the resonance band. However, the ratio $f_{\mathrm{p}} / \Delta f_{\mathrm{p}}$ of the anti-resonance band tends to be equal to the parameters at constant $D$, and especially to the quality factor of the quantity $\left(c_{11}^{\mathrm{p}}\right)^{D}$ in PZ27. This relation of super- $E$ constants with resonance, and super- $D$ constants with anti-resonance, is reminiscent of that which has already been pointed out for the four other resonant geometries in our previous paper [1], but more data would be needed to confirm this point. Actually, in sample PZ35, whose planar Poisson ratio has a relatively important imaginary part, none of the mechanical quality factors approaches the corresponding experimental ratio $f / \Delta f$. neither at resonance nor at anti-resonance, even though the calculated ratios are similar to the experimental values. Perhaps the reason for this is that there are two compliance constants involved in the radial mode, and there may be some as yet unknown mathematical relation between them, related to the widths of the resonance and anti-resonance bands.

\section{Conclusion}

The procedure described in this paper is an extension of our iterative method for the automatic evaluation of piezoelectric ceramics to the case of resonance in the radial mode in lossy materials, with two elastic constants involved, dielectric and piezoelectric, in complex form.

The automatism and accuracy of the previous method are maintained. For the central iteration loop, in which a nonlinear system of equations is solved with the material constants as unknowns, use is made of appropriate mathematical algorithms for the calculation of the modified quotient of Bessel functions in complex form. The same algorithms are used (albeit only as regards real parts) for the determination of the initial values of the two elastic constants. This is equivalent to having the IEEE procedure rigorously automatized, doing away with tables and polynomic approximations, and obtaining both real constants just from the frequencies of the fundamental resonance and its first overtone.

With this method it is possible to characterize materials for which the standard characterization is incomplete, when piezoelectric resonance and antiresonance frequencies are not resolved experimentally, or even when their positions are inverted. The reproduction of all the resonance bands, just by varying 


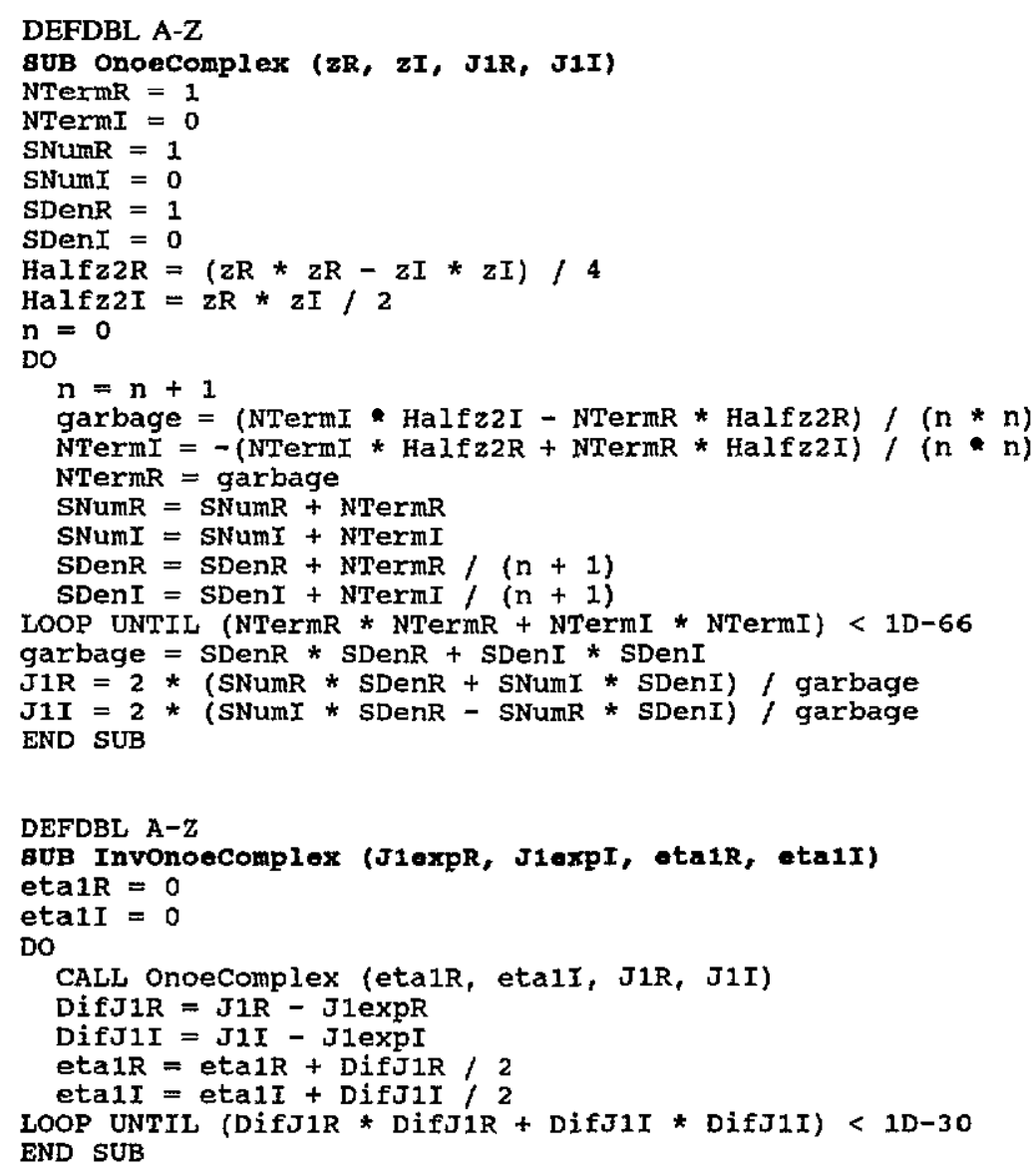

Figure 5. The sub-routines used to calculate Onoe's function and its inverse in QuickBasic.

the frequency in the general expression for the electrical admittance, is excellent for the fundamental resonance, and reasonably good for the first overtone, with small discrepancies that could be ascribed to a frequencydependence of the material constants.

\section{Acknowledgments}

This work has been carried out under project MAT910422 of the Spanish CICYT. We are grateful to Mrs W Wolny, Piezoceramics Division Manager at Ferroperm Ltd, Kvistgård, Denmark for supplying samples.

\section{Appendix}

\section{Direct calculation of Onoe's function $\Im_{1}$}

$\Im_{1}(z)$ is determined at any frequency by first calculating $z$, from equation (3) and substituting in

$\Im_{1}(z)=z \frac{J_{0}(z)}{J_{1}(z)}=z \frac{\sum_{n=0}^{\infty} \frac{(-1)^{n}}{n ! n !}\left(\frac{z}{2}\right)^{2 n}}{\sum_{n=0}^{\infty} \frac{(-1)^{n}}{n !(n+1) !}\left(\frac{z}{2}\right)^{2 n+1}}$

$$
\begin{aligned}
& =2 \frac{\sum_{n=0}^{\infty} \frac{(-1)^{n}}{n ! n !}\left(\frac{z}{2}\right)^{2 n}}{\sum_{n=0}^{\infty} \frac{1}{(n+1)} \frac{(-1)^{n}}{n ! n !}\left(\frac{z}{2}\right)^{2 n}} \\
& =2 \frac{\sum_{n=0}^{\infty} A_{n}}{\sum_{n=0}^{\infty} \frac{1}{(n+1)} A_{n}}
\end{aligned}
$$

where each $A_{n}$, the $n$th term of the sum, is generated from the previous one by

$$
A_{n}=\left[-\frac{1}{n^{2}}\left(\frac{z}{2}\right)^{2}\right] A_{n-1}
$$

and is generally a complex number.

The first term of the sum is $A_{0}=1+i 0$ and the accumulation of terms continues until one is found whose modulus is lower than $10^{-33}$.

\section{Calculation of $c_{11}^{\mathrm{p}}$ from the inverse function of $\Im_{1}$}

If what is needed is the argument $\eta_{1}$ corresponding to Onoe's function $\mathfrak{\Im}_{1}^{\text {(exp) }}=\mathfrak{\Im}_{1 \mathrm{R}}^{\text {(exp) }}-\mathrm{i} \mathfrak{\Im}_{\mathrm{II}}^{\text {(exp) }}$, then an iterative process is undertaken, beginning with $\eta_{1}=0$ because the problem is to find the first solution, which would correspond to the fundamental resonance of the radial mode. With this value, applying equation (A1), a new 
approximation to Onoe's function is generated, which in the $n$th step will be $\Im_{1}^{(n)}=\Im_{1 \mathrm{R}}^{(n)}-\mathrm{i} \mathfrak{\Im}_{1 \mathrm{I}}^{(n)}$.

The iteration will be repeated until $\mid \Im_{1}^{(n)}-$ $\Im_{1}^{\text {(exp) }} \mid<10^{-15}$, increasing the argument by half of the previous difference to ensure convergence, and carefully maintaining the separation between real and imaginary parts. The calculation of $\mathfrak{J}_{1}^{(n)}$ is thus repeated, as many times as necessary, until the convergence criterion is fulfilled; and, once the argument $\eta_{1}$ is known, the determination of $c_{11}^{p}$ by equation (4) is immediate.

The sub-routines by which these calculations in QuickBASIC are made are shown in figure 5. Both the input and output parameters and the variables must be given in double precision.

\section{References}

[1] Alemany C, Pardo L, Jiménez B, Carmona F, Mendiola J and González A M 1994 J. Phys. D: Appl. Phys. 27 148-55

[2] Smits J G 1976 IEEE Trans. Sonics Ultrasonics 23 $393-402$

[3] Holland R 1967 IEEE Trans. Sonics Ultrasonics 14 $18-20$

[4] Meitzler A H, O'Bryan H M Jr and Tiersten H F 1973 IEEE Trans. Sonics Ultrasonics $20233-9$

[5] 1987 IEEE Standard on piezoelectricity ANSI/IEEEStd 176-1987

[6] Sherrit S, Gauthier N, Wiederick H D and Mukherjee B K 1991 Ferroelectrics 119 17-32

[7] Woollett R S and Le Blanc C L 1973 IEEE Trans. Sonics Ultrasonics 20 24-31 